\title{
Target Accrual Number Range
}

National Cancer Institute

\section{Source}

National Cancer Institute. Target Accrual Number Range. NCI Thesaurus. Code C93689.

A range of integers specifying the minimum and maximum number of subjects required. 